\title{
Kernos
}

Revue internationale et pluridisciplinaire de religion grecque antique

$16 \mid 2003$

Varia

\section{G. CASADIo (éd.), Ugo Bianchi. Una vita per la Storia delle Religioni}

\section{André Motte}

\section{OpenEdition}

\section{Journals}

Édition électronique

URL : http://journals.openedition.org/kernos/860

DOI : $10.4000 /$ kernos.860

ISSN : 2034-7871

\section{Éditeur}

Centre international d'étude de la religion grecque antique

Édition imprimée

Date de publication : 1 janvier 2003

Pagination : 390-391

ISSN : 0776-3824

Référence électronique

André Motte, « G. casadıo (éd.), Ugo Bianchi. Una vita per la Storia delle Religioni », Kernos [En ligne], 16। 2003, mis en ligne le 14 avril 2011, consulté le 24 septembre 2020. URL : http://

journals.openedition.org/kernos/860 ; DOI : https://doi.org/10.4000/kernos.860 
son utilisateur à un parcours de lecture, truffé d'extraits et de résumés de travaux sur la religion grecque, et de commentaires plus ou moins critiques selon les cas. Une première partie intitulée "Studiare la religione grecal " s'ouvre avec quelques brèves considérations linguistiques sur la notion grecque de «sacré » (mais sans discuter Rudhardt, ce qui est dommage), passe de L. Gernet et J.-P. Vernant à W. Burkert, W.F. Otto, K. Kerényi et A. Brelich. L'orientation générale de cette première partie relève de l'opposition entre un point de vue fondé sur l'histoire - sur une anthropologie historique - et celui qui postule l'invariance de l'esprit humain dont il recherche les permanences. La deuxième partie («L'espressione religiosa dei Greci delle origini ») se tourne vers la recherche des commencements qui caractérise bon nombre de travaux sur la religion grecque. Balayant d'un revers de plume les postulats du Trattato di Antropologia del sacro dirigé par J. Ries, notamment la contribution de $R$. Boyer sur les indo-européens, il fait le point sur les données religieuses «mycéniennes » avec l'ouvrage déjà ancien de M. Gérard-Rousseau, et une présentation des données par $\mathrm{A}$. Brelich, brève mais remarquable, et les derniers développements avec P. Darcque. Pour cette mise au point, il est clair qu'il faudra désormais tenir compte des dernières découvertes de Thèbes, publiées par V.L. Aravantinos, L. Godart et A. Sacconi ${ }^{1}$. La suite attendue de ces développements sur les « origines »se trouve évidemment dans les travaux de M.P. Nilsson. Tout juste après, un curieux développement sur la prière dans l'Iliade rompt avec l'orientation historiographique adoptée jusque-là. Repartir de la thèse de D. Aubriot sur le sujet (Lyon, 1992) aurait peutêtre permis de conserver la structure expositive de l'ensemble tout en soutenant la volonté de l'A. de se fonder sur un texte ancien déterminé. Le fil historiographique reprend ensuite, avec G. Murray, longuement analysé, A. Brelich et les orientations plus philosophiques de G. Cambiano. La troisième et dernière partie s'intitule « La religione degli Ateniesi. L'interpretazione più recente » et livre en fait une très longue présentation critique (près de 100 pages) de l'ouvrage de R. Parker, Atbenian Religion. A History (Oxford, 1996), avec un petit appendice sur le procès de Socrate analysé par G. De Sanctis. Une bibliographie très fournie referme ce manuel, ainsi qu'un index des noms antiques et des auteurs (modernes) cités. La fin un peu abrupte de l'ouvrage est à l'image de la fin souvent tout aussi abrupte des présentations d'auteurs. Mais sans doute est-ce délibéré : ce parcours est avant tout une mine de réflexions qui donne à penser sur nos propres manières de travailler dans le domaine.

Vinciane Pirenne-Delforge (FNRS - Université de Liège)

Giovanni Casadio (éd.), Ugo Bianchi. Una vita per la Storia delle Religioni, Roma, "Il Calamo", 2002. 1 vol. $17 \times 24$ cm, 524 p. (Biblioteca di Storia delle Religioni, 3). ISBN : 88-88039-24-4.

Sont à l'origine de cet imposant volume deux journées d'étude qui, sous le même intitulé, ont été organisées à l'Université de Salerne, les 14 et 15 avril 1997, à l'initiative de deux disciples, $O$. Bucci et $G$. Casadio. La première de ces journées coïncidait, jour pour jour, avec le deuxième anniversaire du décès d'Ugo Bianchi, né en 1922 et mort inopinément à Rome. Cette manifestation d'hommage était placée sous le patronage de la Société italienne d'histoire des religions, de l'Association internationale pour l'histoire des religions (I.A.H.R.), dont U. Bianchi assurait la présidence au moment de son décès, et du Département des sciences de l'Antiquité de l'Université de Saleme. La vingtaine de contributions qui composent l'ouvrage sont réparties dans trois parties principales. La première, précédée d'une introduction de l'éditeur, est consacrée à la biographie du défunt et évoque plus particulièrement ses activités d'enseignement et de recherche aux Universités de Messine, Bologne et Rome; P. Antes, actuel président de l'I.A.H.R., met en relief sa longue collaboration à cette Association et le rôle important qu'il y joua. L'œuvre d'Ugo Bianchi fait l'objet de la deuxième partie; on y dresse le bilan de ses études portant sur les religions de la Mésopotamie, de l'Iran, de l'Égypte, de la Grèce et de Rome, de ses recherches aussi relatives aussi au christianisme, au gnosticisme, au manichéisme, au

1 Thèbes. Fouilles de la Cadmée I. Les tablettes en Linéaire B de la Odos Pelopidou. Édition et commenlaire, Pisa/Roma, IPE, 2001, spéc. p. 317-325. 
dualisme ainsi qu'à l'ethnologie religieuse. C'est à un examen de la méthode que mettent en œuvre ces travaux, - méthode originale confrontée à celle d'autres maitres illustres, comme R. Pettazzoni et $M$. Eliade, - qu'est consacrée la troisième partie. U. Bianchi, en effet, n'a cessé de s'interroger sur le sens et sur les démarches de la recherche historicoreligieuse. L'hommage unanime qui lui est rendu à ce propos n'empêche pas certains auteurs de prendre à son égard quelque distance critique. Deux articles dont les sujets particuliers ne rentrent pas dans les trois divisions qui précèdent complètent la série des interventions. Suit la bibliographie, très considérable, du savant historien; on la doit à son fils Lorenzo. On peut lire encore, en fin de volume, une vingtaine de messages de sympathie adressés, à l'occasion de la rencontre, par des invités empêchés d'y participer.

La religion grecque occupe une place importante dans cette bibliographie; on y dénombre pas moins de quatre ouvrages ainsi qu'une bonne vingtaine d'articles. G. Casadio leur consacre une étude (Ugo Bianchi e la religione greca, p. 185-200), qui est une version amplifiée de l'hommage paru dans Kernos (9 [1996], p.11-16) et qui reproduit aussi la bibliographie de son maître relative à ce domaine; il souligne notamment le mérite qu'a eu celui-ci de ne pas laisser la religion grecque à l'écart des autres religions du monde antique, il montre l'interêt particulier qu'il nourrissait pour les questions touchant au mysticisme, au syncrétisme, à la sotériologie individuelle, il relève aussi, au passage, des difficultés que soulèvent certaines interprétations. Rappelons pour terminer qu'U. Bianchi fut un ami de la première heure de notre revue : il avait accepté de rédiger un article pour le volume inaugural et de faire partie du comité consultatif. C'est avec émotion et admiration que nous nous souvenons de ce grand historien des religions.

André Motte

(Université de Liège)

\section{Actes de colloques, ouvrages collectifs et mélanges}

Cairns Douglas L. (éd.), Oxford readings in Homer's llias, Oxford, University Press, 2001 [reproduction d'articles publiés antérieurement].

A. LESKr, Divine and buman callsation in Homeric epic, p. 170-202; L.M. SALTKIN, The wratb of Thetis, p. 409-434; M.M. WILLCOCK, Mytbological paradeigma in the Iliad, p. 435-455.

Des Bouvrie Synnøve (éd.), Myth and Symbol I. Symbolic phenomena in ancient Greek culture. Papers from the finst international symposium on symbolism at the University of Tromsø, June 4-7, 1998, Bergen, 2002 (Papers from the Norwegian Institute at Atbens, 5).

Synnøve des Bouvrie, The definition of mytb. Symbolic phenomena in ancient Greek culture, p. 11-69; Herbert Hofrmann, Focitsing the invisible: Greek myth and symbol contemplation, p. 71-88; Jaakko Aronen, Genealogy as a form of mythic discourse: the case of the Pbaeacians, p. 89-111; Stella Georgoud, Gaia/Gê: entre mythe, culte et idéologie, p. 113-134; Jan N. Bremmer, Odysseus versits the Cyclops, p. 135-152; Nanno Marinatos, The Life Cycle of the Arcbaic Greek Warrior and Hero: The Interplay of Myth and Genre in Imagery, p. 153-170; Marjatta Nielsen, Greek myth Etriscan symbol, p. 171-198; Louise Bruit ZaIDMan, Mythe et tragéde dans l'Alceste d'Euripide, p. 199-214; Bruce Lincoln, Retbeorizing Myth, p. 215-232; Dag Øistein EndsJø, To lock up Eleusis: $A$ question of liminal space, p. 233-258; Kirsti K. Simonsuuri, Retbinking Sisyphos, p. 259-274; Jesper Svenbro, Syloson's Cloak and other Greek Mytbs, p. 275-286; Synnøve Des Bouvrie, The Symbol of the Warrior in Greek Tragedy?, p. 287-311; Pierre Eldinger, Artémis, Pan et Maratbon: mythe, polythéisme et évênement bistorique, p. 313-332.

HäGG Robin (éd.), Peloponnesian Sanctuaries and Cults. Proceedings of the Ninth Intemational Symposium at the Swedish Institute at Atbens, 11-13 June 1994, Stockholm, Aströms Förlag, 2002 (ActAth-in $4^{\circ}, 48$ ).

Eleni Konsolaki, A Mycenaean sanctuary on Metbana, p. 25-36; Anastasia Gadolou, Tbe formation of the sacred landscapes of the Eastern Argolid, 900-700 B.C. A religious, social and political survey, p. 37-43; Catherine MORGAN, The Corintbian aristocracy and Corintbian cult during the eighth century $B C$, p. 45-51; Marja C.V. VINK, Sanctuaries and cults in an early urban context: 